\title{
PimT, an amino acid exporter controls polyene production via secretion of the quorum sensing pimaricin-inducer PI-factor in Streptomyces natalensis
}

\author{
Cláudia M Vicente ${ }^{1}$, Javier Santos-Aberturas',2, Susana M Guerra1,2, \\ Tamara D Payero ${ }^{1,2}$, Juan F Martín ${ }^{1,2}$ and Jesús F Aparicio*1,2
}

Address: ${ }^{1}$ Instituto de Biotecnología INBIOTEC, 24006 León, Spain and ²Area de Microbiología, Facultad de Biología, Universidad de León, 24071 León, Spain

Email: Cláudia M Vicente - claudiavicente@netcabo.pt; Javier Santos-Aberturas - jsana@unileon.es; Susana M Guerra - smarg@unileon.es;

Tamara D Payero - tdiep@unileon.es; Juan F Martín - jf.martin@unileon.es; Jesús F Aparicio* - jesus.aparicio@unileon.es

* Corresponding author

Published: 8 June 2009

Microbial Cell Factories 2009, 8:33 doi:10.1 186/1475-2859-8-33
Received: 23 April 2009

Accepted: 8 June 2009

This article is available from: http://www.microbialcellfactories.com/content/8/I/33

(C) 2009 Vicente et al; licensee BioMed Central Ltd.

This is an Open Access article distributed under the terms of the Creative Commons Attribution License (http://creativecommons.org/licenses/by/2.0), which permits unrestricted use, distribution, and reproduction in any medium, provided the original work is properly cited.

\begin{abstract}
Background: Polyenes represent a major class of antifungal agents characterised by the presence of a series of conjugated double bonds in their planar hydroxylated macrolide ring structure. Despite their general interest, very little is known about the factors that modulate their biosynthesis. Among these factors, we have recently discovered a new inducing compound (PIfactor) in the pimaricin producer Streptomyces natalensis, which elicits polyene production in a manner characteristic of quorum sensing. Here, we describe the involvement of an amino-acid exporter from $S$. natalensis in modulating the expression of pimaricin biosynthetic genes via secretion of the quorum-sensing pimaricin-inducer Pl-factor.
\end{abstract}

Results: Adjacent to the pimaricin gene cluster lies a member of the RhtB family of amino-acid exporters. Gene deletion and complementation experiments provided evidence for a role for PimT in the export of L-homoserine, L-serine, and L-homoserine lactone. Expression of the gene was shown to be induced by homoserine and by the quorum-sensing pimaricin-inducer Pl-factor. Interestingly, the mutant displayed $65 \%$ loss of pimaricin production, and also $50 \%$ decrease in the production of $\mathrm{PI}$, indicating that PimT is used as PI-factor exporter, and suggesting that the effect in antifungal production might be due to limited secretion of the inducer.

Conclusion: This report describes the involvement of an amino acid exporter (encoded by pimT in the vicinity of the pimaricin cluster) in modulating the expression of antibiotic biosynthetic genes via secretion of the quorum-sensing pimaricin-inducer PI-factor. The discovery of the participation of amino acid exporters in a signal transduction cascade for the production of polyene macrolides is unexpected, and represents an important step forward towards understanding the regulatory network for polyene regulation. Additionally, this finding constitutes the first detailed characterization of an amino-acid exporter in an Actinomycete, and to our knowledge, the first evidence for the implication of this type of exporters in quorum sensing. 


\section{Background}

Transporters involved in the efflux of low molecular weight substances play important roles in the protection of cells against noxious substances [1], in communication via secretion of regulatory molecules [2], and in the maintenance of an optimal intracellular concentration of metabolites such as sugars [3] and amino acids [4]. Characterization of amino acid efflux systems has been mainly restricted to Escherichia coli and Corynebacterium glutamicum because they are the main bacteria used for amino acid production at the industrial level (see [5] for a review). Work with these species has permitted the molecular characterization of a series of exporters of amino acids in the last few years. These include exporters like RhtB, LysE, ThrE, and YdeD, each representing the prototype of a different transporter family.

The RhtB transporters can be divided into two subfamilies named RhtB and LysE [6], which are structurally very closely related, and resemble each other in molecular mass, and topology of their membrane-spanning helices, showing however slightly different conserved sequence motifs [6]. LysE from C. glutamicum was the first exporter whose gene was cloned [7], and exports basic amino acids like L-lysine and L-arginine at comparable rates [8]. In E. coli, RhtB confers resistance to L-homoserine, and Lhomoserine lactone, whereas RhtC drives the efflux of Lthreonine [9]. YfiK and YeaS from E. coli also belong to the RhtB family of export proteins, and promote the export of L-cysteine and O-acetylserine [10], and L-leucine [11], respectively.

The C. glutamicum ThrE exporter does not belong to the RhtB superfamily: it is larger in size, it shows ten transmembrane-spanning helices [12], and it exports both Lthreonine and L-serine [13], whereas the E. coli exporter YdeD is implicated in the export of O-acetylserine or Lcysteine and belongs to the PecM family of transporters [14].

Bacteria belonging to the genus Streptomyces are wellknown for their ability to produce a variety of antibiotics and other secondary metabolites [15]. Production of these compounds is regulated in response to an altered nutritional status [16] and to a variety of environmental conditions, and hence occurs in a growth-phase-dependent manner and usually accompanied by morphological differentiation [17]. Pimaricin is a tetraene macrolide antifungal antibiotic produced by $S$. natalensis. As a polyene, its antifungal activity lies in its interaction with membrane sterols, which alters the membrane structure and leads to the leakage of cellular materials [18]. Like other macrocyclic polyketides, pimaricin is synthesized by the action of so-called type I modular polyketide synthases [19]. Its biosynthetic gene cluster has been characterized
[20-27], and some of the factors that modulate its biosynthesis have been identified [28].

Secondary metabolism and cell differentiation in actinomycetes are often controlled by diffusible butyrolactones which act as quorum-sensing signals [29]. Recently, we have identified a novel quorum-sensing inducer (PI-factor; 2,3-diamino -2,3-bis (hydroxymethyl) -1,4-butanediol) which elicits pimaricin production at nanomolar concentrations [30]. Here, we describe for the first time, the cloning, sequencing and detailed characterization of an amino-acid exporter from a Streptomycete, and demonstrate its role as a PI-factor transporter in the pimaricinproducing S. natalensis.

\section{Results \\ Cloning of pimT}

pimT was identified by genomic walking using an $S$. natalensis ATCC 27448 cosmid library [20] and DNA segments from pimM (which encodes a pathway-specific regulatory gene for pimaricin production [26]), at the left hand of the pimaricin gene cluster [21]. The gene was sequenced from plasmid pCMV01 (see Methods) and turned out to be separated by 1,366 bp from the 3' end of $\operatorname{pim} M$, and in the same orientation (Fig. 1). The initiating ATG codon of pimT is preceded by the sequence AGGAGG which could potentially act as a ribosomal binding site. pimT is 645 bp long with an overall codon usage pattern in good agreement with that of typical Streptomyces genes.

\section{In silico analysis of the pimT gene product}

Computer-assisted analysis of the pimT gene product (214 amino acids with an estimated $M_{r}$ of 22,248) showed a high sequence identity ( $87.9 \%)$ with the whole of protein Orf16 of Streptomyces hygroscopicus NRRL 3602, a putative RhtB protein of 227 amino acid residues whose encoding gene was found within the geldanamycin gene cluster [31], and also with SACE 5283 (57.2\% identity), a putative LysE protein encoded by the Saccharopolyspora erythraea NRRL 2338 genome [32]. Protein database comparisons revealed several additional counterparts, all of them putative RhtB proteins. Interestingly, no homologues were found to be encoded by the Streptomyces genomes sequenced up to date, including $S$. coelicolor, $S$. avermitilis, S. scabies or S. griseus, suggesting that PimT is probably involved in processes related to strain-specific secondary metabolism. PimT analysis revealed that it is highly hydrophobic; it contains six predicted transmembrane helices, and the three conserved sequence signatures of RhtB proteins [6] situated at canonical distances.

\section{Gene replacement of pimT}

In order to determine the function of pimT, we inactivated it by using the REDIRECT gene replacement technology as indicated in Methods. Double-crossover mutants were 

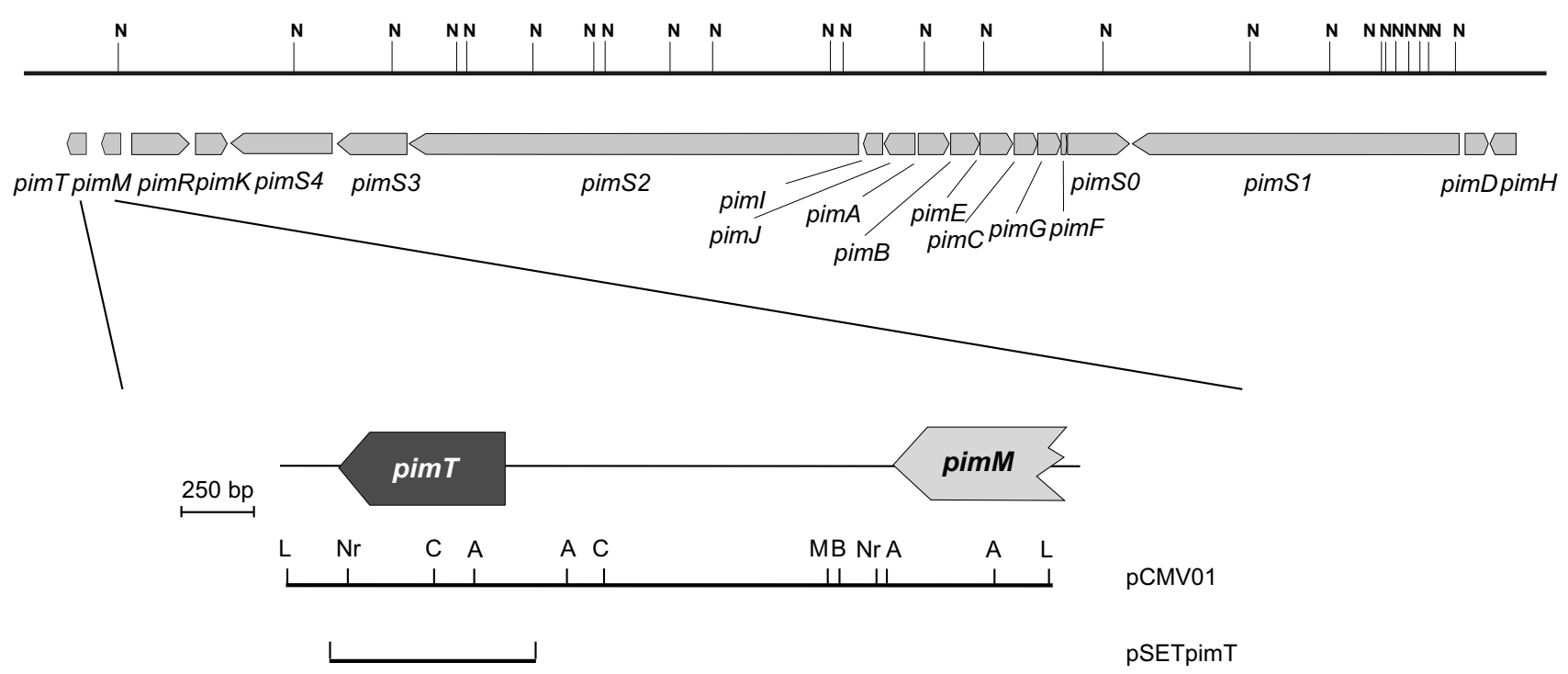

\section{Figure I}

Pimaricin biosynthetic gene cluster. The left fringe of the gene cluster is indicated in more detail, and includes pimT (in dark grey), and the 3'-end of the transcriptional activator-encoding gene for pimaricin biosynthesis pimM [26]. Pointed boxes indicate the direction of transcription. A, Apal; B, BamHI; C,Accl; L, ApaLI; M, Mlul; N, Notl; Nr, Nrul. The inserts used for vector construction are indicated at the bottom.

screened by apramycin resistance and kanamycin sensitivity (Fig. 2). These (about 1\%) were verified by both PCR and Southern blot analysis. Fig 2 shows the Southern blot of a randomly chosen exconjugant DNA. Chromosomal DNAs isolated from S. natalensis ATCC 27448 and mutant $\triangle p i m T$ and digested with AflIII were probed with a 809 bp $P v u$ I fragment covering the whole of pimT (Fig. 2A). A hybridizing band of $2.9 \mathrm{~kb}$ was found for the wild type as expected (Fig. 2B), whereas in the mutant, two hybridizing bands of $2.2 \mathrm{~kb}$ and $1.5 \mathrm{~kb}$ were observed (Fig. 2B), indicating that a double crossover event had occurred. The observed hybridizing bands corresponded exactly to those expected according to the integration process depicted in Fig. 2.

The new strain S. natalensis $\Delta p i m T$ had growth and morphological characteristics identical to those of $S$. natalensis wild type when grown on solid or liquid media, suggesting that PimT has no role in bacterial growth or differentiation. The spore counts of both strains were similar after growth for 9 days at $30^{\circ} \mathrm{C}$ on TBO plates. The spores of both strains were serially diluted and plated on minimal medium. Both strains grew well in the minimal medium, showing an identical growth curve, which indicates that genes involved in amino acid biosynthesis were not affected.

\section{$\Delta$ pimT mutant shows increased sensitivity to $L$ - homoserine, L-serine, and L-homoserine lactone}

Some natural amino acids are known to inhibit $E$. coli cell growth when added into minimal media at high concentrations [9,33], and the same phenomenon has been observed in several Streptomyces strains (unpublished results). Therefore, the involvement of PimT in the export of amino acids was tested by comparing the resistance of the wild-type strain and the null-mutant for the pim $T$ gene to various natural amino acids (Table 1). The $\Delta$ pimT mutant was found to have increased sensitivity to Lhomoserine (3-fold), L-serine (2-fold) and L-homoserine lactone (1.6-fold) (Table 1). These data suggest that protein PimT assists the export of L-homoserine, L-serine, and L-homoserine lactone out of the cell. No differences in sensitivity were observed when D-homoserine was tested, thus indicating that PimT exhibits stereospecificity for Lhomoserine. Also, no differences were observed when other amino acids were tested (Table 1).

Direct measurements of intracellular or extracellular concentrations of PimT amino acid substrates in the wild type and the mutant yielded no detectable differences between the two strains, probably because $S$. natalensis is not an overproducer.

\section{Transcriptional initiation site and expression of pimT}

To define the transcript initiation site of pimT, $143 \mathrm{bp}$ DNA fragment upstream from the ATG start codon was 
A)

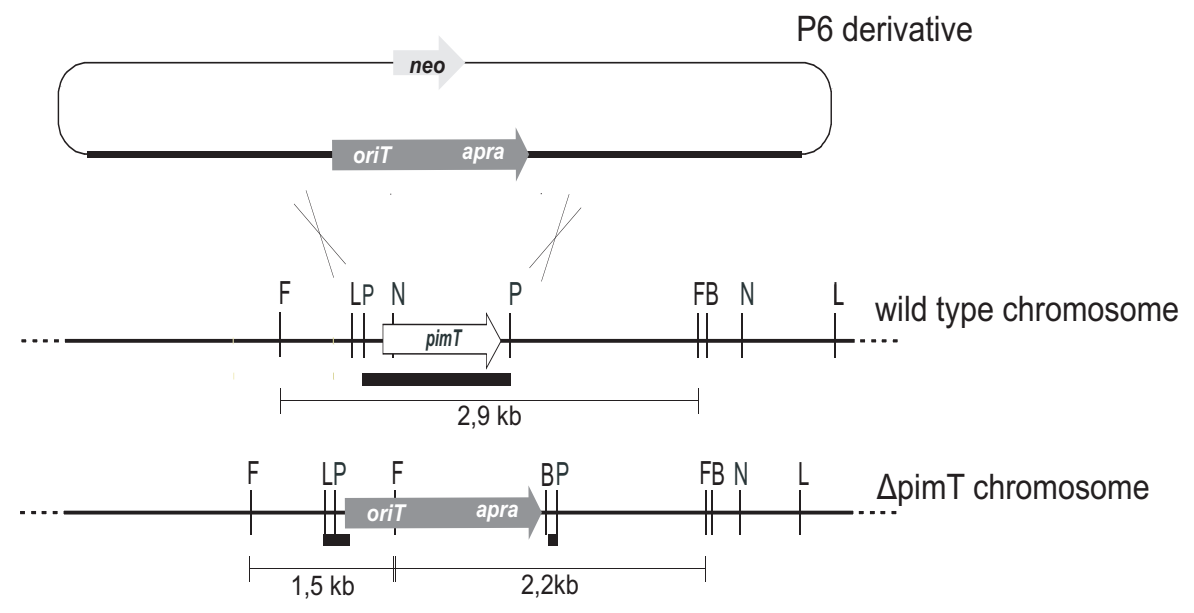

B)

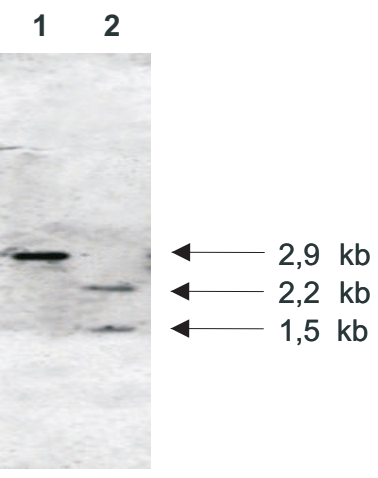

\section{Figure 2}

Gene replacement of pimT. A) Predicted restriction enzyme polymorphism caused by gene replacement. The Aflll restriction pattern before and after replacement is shown. The probe is indicated by thick lines. B, BamHI; F, Aflll; L, ApaLl; N, Nrul; P, Pvul. B) Southern hybridization of the Aflll digested chromosomal DNA of the wild type (lane I), and $\Delta$ pimT (lane 2) strains.

amplified and cloned into the promoter-probe vector pIJ4083 to yield pPpimT (see Methods). This plasmid was used to transform S. lividans, and catechol 2,3-dioxygenase activity was measured after growth for several time periods. Expression in YEME medium reached a maximum at $27 \mathrm{~h}$ (290 $\mathrm{mU} / \mathrm{mg}$ protein) and decreased thereafter (Fig. 3A). The same S. lividans strain transformed with pIJ4083 (control) yielded no catechol dioxygenase activity. Also, no promoter activity could be detected when we used the successive 152 bp DNA fragment immediately upstream (not shown). The origin of transcription of the pimT promoter was determined by several primer extension experiments using a carboxyfluorescein labeled primer (see Methods). These revealed a single transcription start point at 29-30 nucleotides upstream of the ATG translation start site (Fig. 3C). Analysis of the region upstream of the transcription starting site revealed the presence of a -10 box TTGTAT, located at 6 nucleotides from the start site, and a -35 box GTGCCG separated by 16 nucleotides (Fig 3D).

\section{pimT expression is induced by homoserine}

It is known that expression of some genes encoding amino acid transporters is induced by their corresponding substrates $[8,11]$. In order to ascertain whether pimT expression was induced by its substrates, we added different concentrations of either L-homoserine, L-serine, or Lhomoserine lactone to pPpimT-containing S. lividans cultures, and XylE activity of crude extracts was monitored at different time points. These data clearly showed that pimT 
Table I: Resistance of S. natalensis strains to some amino acids.

\begin{tabular}{lccc}
\hline Amino acid & \multicolumn{3}{c}{ Minimal inhibitory concentration $(\mu \mathrm{g} / \mathrm{ml})$} \\
\hline & $w t$ & $\Delta$ pimT & $\Delta$ pimT $($ pSETpimT $)$ \\
\cline { 2 - 4 } & & & \\
L-Aspartic acid & 5000 & $>5000$ & \\
L-Methionine & $>5000$ & $>5000$ & \\
L-Threonine & $>5000$ & $>5000$ & \\
L-Lysine & $>5000$ & $>5000$ & 75 \\
DL-Hydroxynorvaline & 180 & 180 & \\
L-Homoserine & 60 & 20 & 81 \\
D-Homoserine & $>200$ & $>200$ & 9000 \\
L-Homoserine lactone & 110 & 70 & \\
L-Serine & 7400 & 3700 & \\
$\gamma$-Butyrolactone & $>5000$ & $>5000$ & \\
\hline
\end{tabular}

expression was increased substantially by the addition of homoserine $(2 \mathrm{mg} / \mathrm{ml})$ to the growth medium (Fig. 3A), whereas no differences were observed when we added serine or homoserine lactone (not shown). The increase in expression ranged between 3.1 -fold at $33 \mathrm{~h}$ of growth to 2.1 -fold at $48 \mathrm{~h}$. Also, the expression profile changed when compared with the control culture, reaching its maximum after a longer incubation period ( $33 \mathrm{~h}$ ) (Fig. 3A).

To investigate this further, pimT expression in the presence and absence of added homoserine was examined in cultures of the $S$. natalensis wild-type strain. Total RNA was prepared from $S$. natalensis wild type after growth for $48 \mathrm{~h}$ in YEME medium, in the presence or absence of amino acid $(2 \mathrm{mg} / \mathrm{ml})$, and used as template for gene expression analysis by reverse transcriptase-polymerase chain reaction (RT-PCR) as described in the Methods section. Primers for RT-PCR were designed to produce cDNAs of approximately $500 \mathrm{bp}$. A primer pair designed to amplify a cDNA of the lysA gene (encoding diaminopimelate decarboxylase) was used as an internal control. Transcripts were analyzed after 28 and 30 PCR cycles, and analyses were carried out three times for each primer pair. There was a clear increase in the expression level of pimT in the presence of added homoserine after both 28 and 30 PCR cycles (Fig. 3B), again consistent with the induction of pimT expression by L-homoserine.

\section{Deletion of pimT reduces pimaricin production}

The fermentation broth produced by the mutant strain generated by gene replacement, $S$. natalensis $\Delta$ pimT, was extracted with butanol and analyzed for the presence of pimaricin. The high performance liquid chromatography (HPLC) analysis indicated that pimaricin production in the mutant strain $\Delta p i m T$, was only about $35 \%$ of the pimaricin accumulated by the wild type strain at $96 \mathrm{~h}(0.73 \mathrm{~g} /$ l) (Fig. 4A). Given that both strains showed identical growth curves, this result prompted further investigation of the possible reasons for the markedly lower pimaricin production.

\section{$\triangle$ pimT mutants sustain low production of the quorum- sensing pimaricin-inducer Pl-factor}

Given that the majority of members of the RhtB and LysE families export small positively-charged molecules, and that it has been suggested that their physiological role might be to avoid the build-up of substrate compounds to toxic levels in the cytoplasm or to mediate the secretion of signalling molecules $[5,6,34]$, we decided to investigate the involvement of PimT in the export of the quorumsensing pimaricin inducer PI-factor [30]. PI factor $(2,3-$ diamino-2,3-bis(hydroxymethyl)-1,4-butanediol) (Fig. 5 ) is a positively charged molecule which might well be an additional substrate for PimT. To analyze the putative involvement of PimT in the efflux of PI factor, S. natalensis $\triangle$ pimT was grown in YEME medium, and extracellular PI factor production was monitored. Only about $50 \%$ of the PI factor produced by the wild type could be detected in $S$. natalensis $\Delta$ pimT cultures after $48 \mathrm{~h}$ of growth (Fig. 5). This result suggests that PimT plays a role in PI factor export, and that the levels of PI observed might be due in part to diffusion or to another as yet unidentified carrier. Interestingly, deletion of pimT gene increased the intracellular pool of PI. These data (calculated as the mean from three independent experiments) were, respectively, 0.26 and $0.45 \mathrm{nmol} / \mathrm{mg}$ dry weight in the wild type strain and the mutant after $48 \mathrm{~h}$ of growth. This result indicates that PimT protein influences the accumulation of PI-factor in the medium rather than the synthesis of this inducer in the cell, and strongly suggests that the PI signal must be sensed at the surface of the cell.

\section{Addition of exogenous PI-factor restores pimaricin production in $\triangle$ pimT mutants}

In order to ascertain whether the reduction of pimaricin production observed in the mutant was due to the substantial decrease in PI-factor export, purified PI-factor was added exogenously to cultures of $\triangle p i m T$ mutants, and antifungal production was monitored. Cells were grown in YEME medium in the presence and absence of $100 \mathrm{nM}$ PI-factor and pimaricin production was assessed. It was found that polyene production in the supplemented cultures was restored to wild type levels (not shown), thus corroborating the role of PimT as PI-factor exporter, and indicating that the extracellular concentration of PI-factor is crucial for pimaricin production.

\section{pimT expression is also induced by $\mathrm{Pl}$-factor}

In order to test whether pimT expression was induced by PI-factor, we added different concentrations of it to pPpimT-containing S. lividans cultures, and XylE activity of crude extracts was monitored at different time points. 
A)

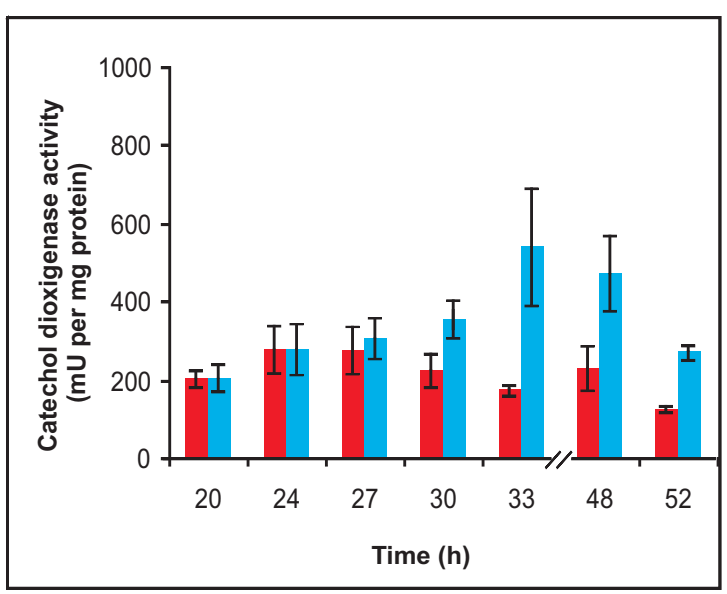

B)
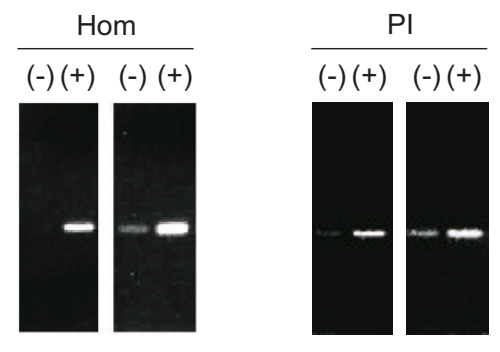

$(-)(+)$

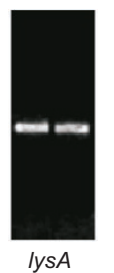

C)
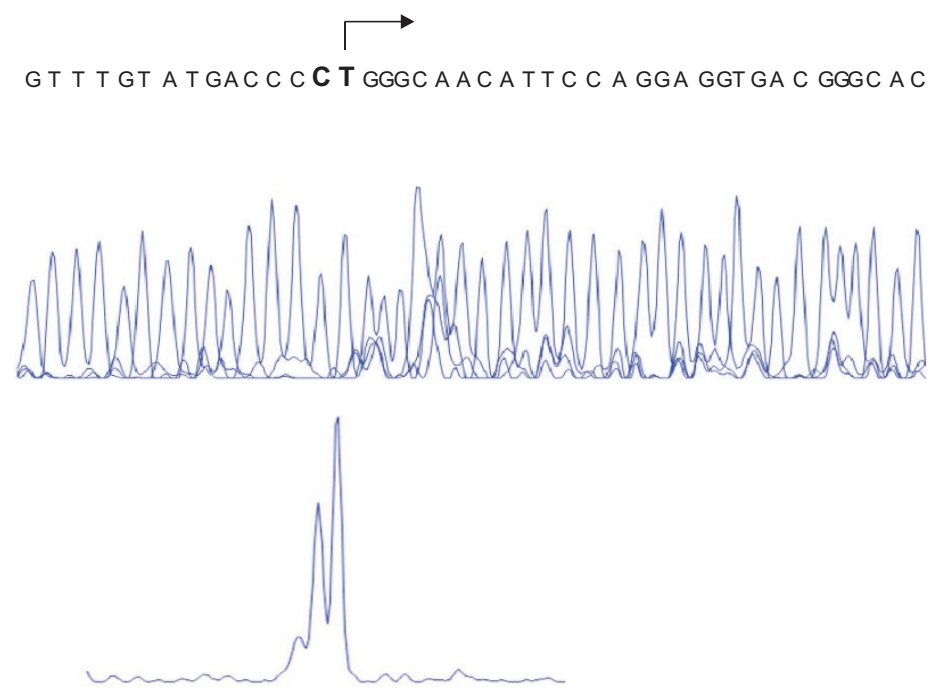

D)

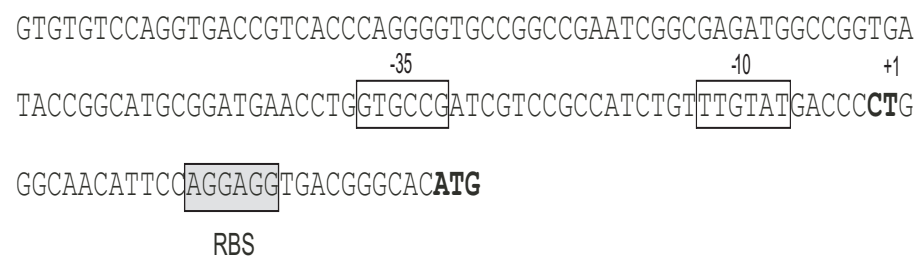

Figure 3 (see legend on next page) 
Figure 3 (see previous page)

Promoter activity, gene expression and primer extension analyses. A) Time course of promoter activity of pimT using the $x y I E$ gene (encoding catechol dioxygenase) as reporter. The enzyme activity of crude extracts was measured at the indicated culture times. Cells were grown in the presence (blue) or absence (red) of added homoserine (2 mg/ml). B) Gene expression analysis of pimT by RT-PCR. Analysis was carried out on S. natalensis wild type strain in the presence $(+)$ or absence (-) of added homoserine or PI-factor as indicated in the Methods section after 28 (left panels) and 30 (right panels) PCR amplification cycles. The identity of each amplified product was corroborated by direct sequencing. The absence of contaminating DNA in the RNA samples was assessed by PCR. Transcription of the lys gene (encoding diaminopimelate decarboxylase) located outside the pim cluster was also assessed as an internal control. The result of the analysis in the absence (-) or presence (+) of homoserine after 30 PCR cycles is shown. Identical result was observed in the presence of PI-factor. C) Mapping of the transcriptional start site of pimT by primer extension analysis. The full nucleotide sequence is shown in the upper fluorogram. The nucleotides corresponding to the end of the primer extension are highlighted. The arrow shows the direction of transcription. In the lower panel (D), the start of the transcript is indicated in bold. The -10 and -35 hexanucleotides are boxed, and the first codon is shown in boldface letters. Nucleotides showing homology with the I6S RNA that could form a ribosomal binding site are framed with a box labelled RBS.

No increase in expression was observed at all (not shown), which is consistent with the signaling role of PIfactor at the membrane level in S. natalensis. However, when we studied pimT expression in the presence and absence of added PI-factor in cultures of $S$. natalensis wildtype strain by RT-PCR (see above), results showed a clear difference in the expression level of pimT in the presence or absence of the inducer after both 28 and 30 PCR cycles (Fig. 3B), being clearly higher in the presence of PI-factor, thus suggesting that pimT expression is also induced by PIfactor.

\section{Gene complementation restores wild type phenotypes}

To confirm that the disruption of pimT was directly responsible for the reduction of pimaricin production, we complemented the pimT-disrupted mutant with pimT. A DNA fragment containing pimT plus its promoter region was inserted into the integrative vector pSET152neo, giving rise to pSETpimT (see Methods). The plasmid was transferred from E. coli ET12567 [pUZ8002] to S. natalensis $\Delta$ pimT by conjugation. pSET152neo was also introduced into $S$. natalensis wild type as control. Introduction of pSETpimT restored pimaricin biosynthesis to the control levels (Fig. 4B). These results were fully consistent with those obtained upon deletion of the pimT gene, and confirm the involvement of PimT in pimaricin biosynthesis.

It is noteworthy that production of pimaricin diminished substantially upon introduction of pSET152neo, reaching about $44 \%$ of the production observed in the wild type strain without plasmid (Fig. 4A). Similarly, production of the complemented strain, although identical to that of the control strain, was lower than that of the wild type strain bearing no plasmid. Furthermore, introduction of pSET152neo into the mutant also reduced severely pimaricin production, reaching about $25 \%$ of the production observed in the mutant strain without plasmid (Fig. 4).
This phenomenon is well known in many Streptomyces species, and is thought to be derived from the integration of the plasmid into the $\Phi \mathrm{C} 31 \mathrm{attB}$ site. It has been reported that integration of vectors with an attP-int locus from $\Phi C 31$ can cause detrimental effects on antibiotic production in some strains [35], and this could be the case of pimaricin and $S$. natalensis. Moreover, it has been reported that pSET152 vectors can integrate into pseudoattB sites in both $S$. coelicolor and S. lividans [36] and this could also be happening in $S$. natalensis, thus affecting pimaricin production.

Interestingly, gene complementation also restored PI-factor production to wild-type levels thus confirming the role of the gene product in PI factor secretion. Similarly, the complemented strain also recovered the resistance levels of the wild type to L-homoserine, L-serine, and L-homoserine lactone (Table 1).

\section{Discussion}

Sequencing of the left-hand side of the pimaricin gene cluster in $S$. natalensis revealed the presence of a gene, pimT, whose product was found to display six predicted transmembrane helices and was strikingly similar to proteins of the RhtB family of amino acid exporters. Members of this family of exporters are widespread in eubacteria and archaea [6], and most prokaryotic genomes contain a significant number of genes devoted to them [37], but despite their interest only a few have so far been studied in detail. The members of this family that have been characterized mostly belong to C. glutamicum or E. coli [9$11,33]$, most likely because these are the two species used in the industrial production of amino acids [34]. Strikingly, no homologues were found to be encoded by any of the Streptomyces spp. genomes that have been sequenced up to date. This fact is unexpected for a gene whose proposed function is to achieve homeostasis of the intracellular amino acid concentration, and suggests that the 
A)
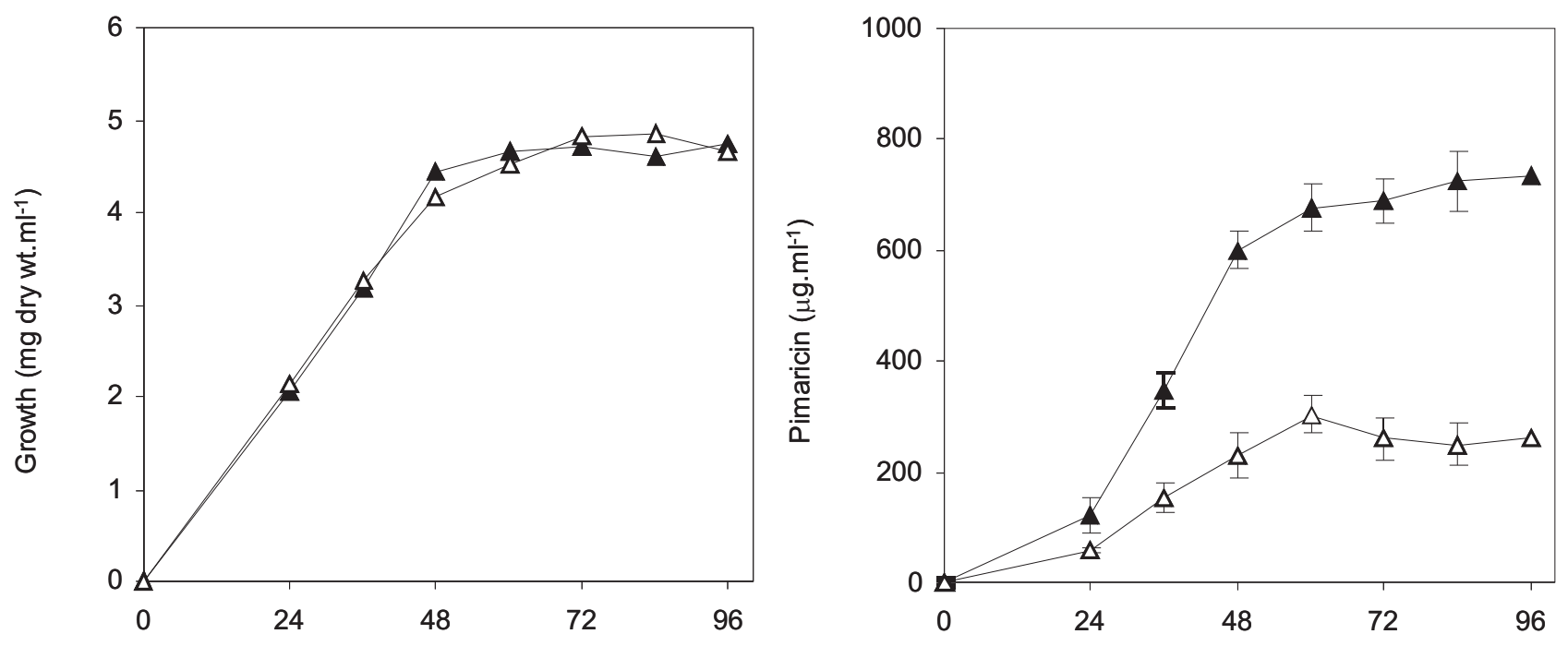

\section{B)}
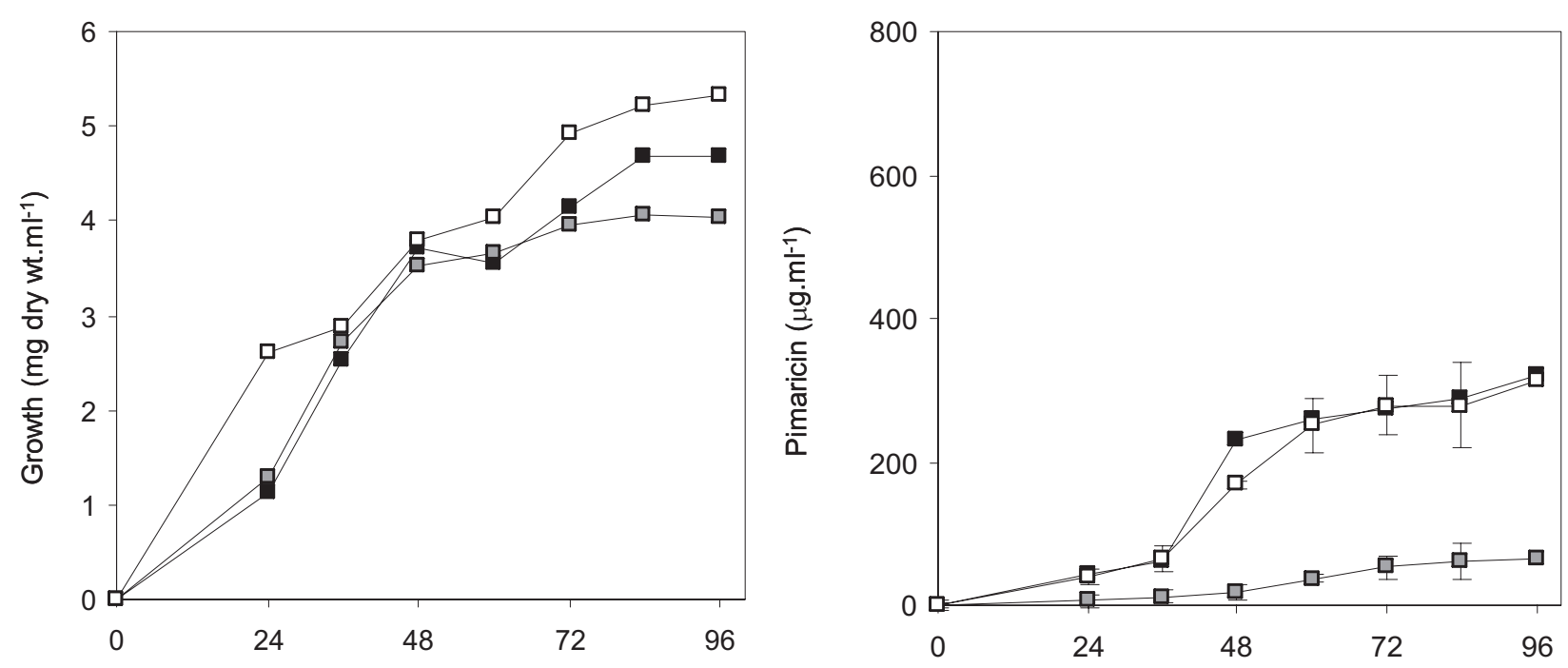

Figure 4

Replacement of PimT reduces pimaricin production and gene complementation restores antifungal biosynthesis. Production of pimaricin in YEME medium (right panels). A) solid triangles indicate production by the wild type strain, open triangles the production by the $\Delta$ pimT strain. B) black squares indicate the production by the wild type strain harboring PSETI52neo (control), white squares the production by the $\Delta$ pimT strain complemented with pimT, and grey squares the production by the $\triangle$ pimT strain harboring PSETI52neo. Data are the average of three triplicate flasks. Vertical bars indicate the standard deviation values. Growth curves are shown at the left panels. 


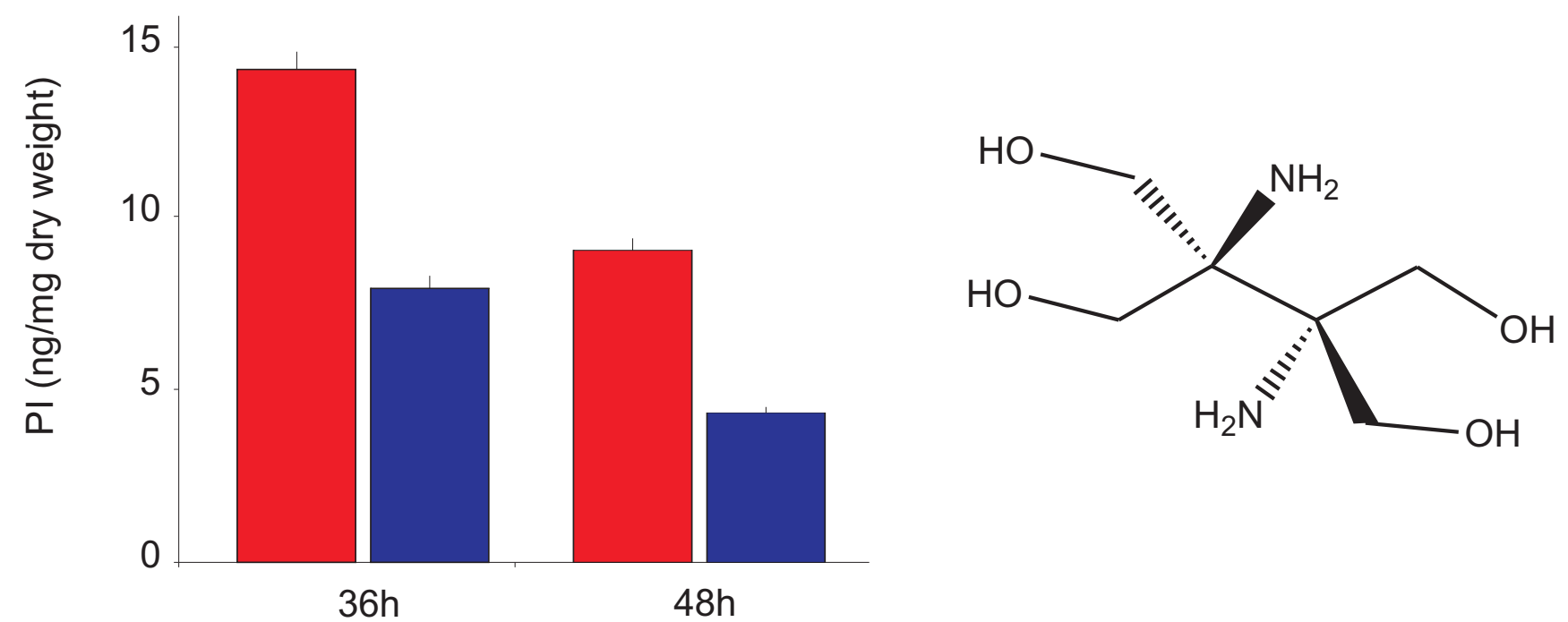

\section{Figure 5}

PI factor production is reduced in the mutant. Detection was carried out after $36 \mathrm{~h}$ and $48 \mathrm{~h}$ of growth at $250 \mathrm{rpm}$ and $30^{\circ} \mathrm{C}$ in YEME medium. Red bars indicate production by the wild type strain, blue bars the production by the $\Delta$ pim $T$ strain. Pure PI factor was used as standard. Data are the average of three flasks. Error bars indicate the standard deviation values. The structure of the PI factor is indicated.

biological function of this gene may not be restricted only to amino acid transport and might be related to strainspecific secondary metabolite biosynthesis and regulation. The lack of studies in Streptomyces spp. prompted us to study the involvement of this gene product in the export of amino acids and/or secondary metabolism in $S$. natalensis.

Comparison of the resistance of the wild-type, the $\Delta$ pimT strain, and the complemented strain to various amino acids provided evidence for a role of pim $T$ in the excretion of L-homoserine, L-serine, and L-homoserine lactone. In Gram negative bacteria $\mathrm{N}$-acyl-homoserine lactones serve as signals that mediate cell to cell communication in concert with cell density [38]. Such quorum sensing systems regulate a variety of global cellular response processes such as, e.g., bioluminescence in Vibrio, or virulence in Pseudomonas aeruginosa and other pathogens, among others (see [39] for a review). Interestingly, homologues of PimT such as RhtB, have been described to be involved in the efflux of homoserine lactone in E. coli [9] and based on this their implication in quorum sensing has been suggested [37]. Our present data demonstrate the involvement of an RhtB amino acid exporter in quorum sensing through the secretion of not only homoserine lactone, but also the quorum sensing pimaricin-inducer PI-factor [30]. This factor triggers pimaricin production in S. natalensis mutants that had lost their ability to produce pimaricin in a manner characteristic of quorum sensing [30]. A factor, a well-known S. griseus autoregulator of the butyrolactone class [40], has the same effect on S. natalensis [30], but whereas A-factor recognition has been described to take place intracellularly [40], our results indicate that PI-factor must be recognized at the membrane level. Given that S. natalensis is unable to synthesize A-factor [30], it appears that this strain is able to integrate foreign quorum signals. Moreover, the existence of a gene in S. natalensis encoding a butyrolactone-receptor protein [41] has recently been reported. This sensing of "foreign" quorumsensing signals might be a general phenomenon in bacteria, since E. coli is able to respond to different quorumsensing signals without producing them [42].

The substantial decrease of PI factor release upon deletion of the pimT gene explains the low pimaricin production observed in the mutant strain. Conversely, the complemented strain shows a recovery in the production of extracellular PI factor and also of pimaricin, thus suggesting that the PI signal must be sensed at the surface of the cell. Taken together, these results constitute strong evidence for the implication of PimT in the efflux of the inducer. Interestingly, the S. natalensis $\Delta p i m T$ mutant still excreted some PI factor out of the cell. Such efflux might be due to diffusion, although the participation of other carriers cannot be excluded.

As already indicated (see above) the detrimental effect on pimaricin production observed upon introduction of the pSET152-derived vector used in this study could be explained by the use of the attP-int locus from $\Phi C 31$ 
[35,36]. In this regard, we have also observed that introduction of PSET152-derived vectors into the model Streptomycete $S$. coelicolor $\mathrm{A} 3(2)$ causes a reduction in the production of the polyketide antibiotic actinorhodin (unpublished results). Along with this, the use of vector pTO1, an integrative plasmid which uses the integrative functions of phage $\Phi С 31$, has also been described to reduce the production of bialaphos in the producing strain S. hygroscopicus ATCC 21705 [43]. Thus, it seems that this phenomenon is not specific to $S$. natalensis and pimaricin. Hence, it is conceivable that the use of vectors with different integrative functions will overcome such problem. Bearing these results in mind, it is likely that the reported increment in pimaricin production upon gene duplication of the PAS regulator pimM in the S. natalensis wild type strain by means of the pSET152-derived vector pSETpimM [26] is actually underestimated, and the actual effect of gene duplication could well be substantially higher than that previously reported by us.

The proteins of the RhtB family are among the most widespread membrane proteins. Most prokaryotic genomes contain a significant number of genes (up to eighteen) devoted to them [37]. As examples, E. coli has as many as five paralogues [5], whereas Saccharopolyspora erythraea, a Gram positive bacterium, has ten [32]. This fact gives an idea of the importance of the physiological role of these proteins for the cell. However, the significance of the excretion of amino acids is still unclear. Most of the RhtB exporters studied are characterized by relatively wide substrate specificity, and this aspect led to the suggestion that amino acids are accidental substrates for these proteins $[5,8]$. Whether these proteins export amino acids because of the low specificity of these carriers for their true substrates [8] or because their actual substrates are the amino acids they transport [37] is a matter of debate. In any case, it has been suggested that these systems might be involved in quorum sensing [6], and the role of PimT as an exporter of the quorum sensing pimaricin-inducer PI-factor provides clear evidence for this idea.

\section{Conclusion}

Polyenes represent a major class of antifungal agents characterised by the presence of a series of conjugated double bonds in their planar hydroxylated macrolide ring structure. Despite their general interest, very little is known about the factors that modulate their biosynthesis. This report describes the involvement of an amino acid exporter (encoded by pim T in the vicinity of the pimaricin cluster) in modulating the expression of antibiotic biosynthetic genes via secretion of the quorum-sensing pimaricin-inducer PI-factor. The discovery of the participation of amino acid exporters in a signal transduction cascade for the production of polyene macrolides is unexpected, and represents an important step forward towards under- standing the regulatory network for polyene regulation. Additionally, to our knowledge, this finding provides the first evidence for the implication of this type of exporters in quorum sensing.

\section{Methods \\ Bacterial strains, cloning vectors and cultivation}

S. natalensis ATCC 27448 was routinely grown in YEME medium [44] without sucrose. Sporulation was achieved in TBO medium [20]. For pimaricin and PI-factor production, the strains were grown in YEME without sucrose at $30^{\circ} \mathrm{C}$ and $250 \mathrm{rpm}$. Escherichia coli strain XL1-Blue MR (Stratagene) was used as a host for plasmid subcloning in plasmids pBluescript (Stratagene), pUC18 and pUC19. E. coli ET12567 [pUZ8002] was used as donor in intergeneric conjugations. E. coli BW25113 was used as the host for Red recombination [45] and to propagate plasmid pIJ790 [46]. S. lividans 66 [44] was used for promoter activity assessment. The promoter-probe vector used was pIJ4083 which contains a promoterless $x y l E$ gene [44]. pSETneo is a pSET152 [47] derivative constructed as follows: pTC192-km [48] was digested with DraI and Ecl136II and religated to yield pMB1neo; this plasmid was subsequently digested with EcoRI, end-filled with Klenow, and religated to eliminate the unique EcoRI site, and then cut with BamHI to generate a 913 bp fragment containing the neo resistance gene which was cloned into the Bam HI site of pSET152. Minimal inhibitory concentrations (MICs) of amino acids were determined on Streptomyces MM agar plates [44] containing different concentrations of amino acid. The plates were spotted with 200 viable spores and the MIC was determined after 5 days incubation at $30^{\circ} \mathrm{C}$.

\section{Genetic procedures}

Standard genetic techniques with E. coli and in vitro DNA manipulations were as described by Sambrook and Russell [49]. Recombinant DNA techniques in Streptomyces species and isolation of Streptomyces total DNA were performed as previously described [44]. Southern hybridization was carried out with probes labeled with digoxigenin by using the DIG DNA labeling kit (Roche Biochemicals). Intergeneric conjugation between E. coli ET12567 [pUZ8002] and S. natalensis was performed as described [50].

\section{DNA sequencing and analysis}

A 2,661 bp ApaLI fragment encompassing the entire pimT gene, and the 3'-terminal end of the pimM gene (Fig. 1) was blunt-ended with Klenow and cloned into a SmaI-cut pUC19 vector to yield pCMV01. This plasmid was then used as a source of DNA for sequencing. Sequencing templates were obtained by random subcloning of fragments generated by controlled partial HaeIII digestions. DNA sequencing was accomplished by the dideoxynucleotide 
chain-termination method using the Perkin Elmer Amplitaq Gold Big Dye-terminator sequencing system on double-stranded DNA templates with an Applied Biosystems ABI 3130 DNA genetic analyzer (Foster City, California, USA). Each nucleotide was sequenced a minimum of three times on both strands. Alignment of sequence contigs was performed using the DNA Star program Seqman (Madison, Wis.). DNA and protein sequences were analyzed with the NCBI World Wide Web BLAST server.

\section{Construction of a $\Delta$ pimT mutant}

Deletion of pimT of $S$. natalensis was made by replacing the wild-type gene with a cassette containing an apramycin selective marker using a PCR based system [46]. The plasmid pIJ773 containing the apramycin resistance gene (aac(3)IV) and the oriT replication origin was used as a template. The mutant was constructed using the oligonucleotides 5'-tgaccgctgggcaagattccaggaggtgacgggcacatgATTCCGGGGATCCGTCGACC-3' and 5'ggcgccgcgacgccgcggagatccgtcagaagcgccctaTGTAGGCT-

GGAGCTGCTTC-3' as the forward and reverse primers respectively (the sequence identical to the DNA segment upstream from the start codon of pimT is in bold and in lower case and the sequence identical to the segment downstream from the stop codon of pimT is in lower case italics). These two long PCR primers (59 nt and $58 \mathrm{nt}$ ) were designed to produce a deletion of pim $T$ just after its start codon leaving only its stop codon behind. The 3' sequence of each primer matches the right or left end of the disruption cassette (the sequence is shown uppercase in both primers). The extended resistance cassette was amplified by PCR and E. coli BW25113/pIJ790 bearing cosmid P6 [20] was electro-transformed with this cassette. The isolated mutant cosmid was introduced into nonmethylating E. coli ET12567 containing the RP4 derivative pUZ8002. The mutant cosmid was then transferred to $S$. natalensis by intergeneric conjugation [50]. Double crossover exconjugants were screened for their kanamycin sensitivity and apramycin resistance.

\section{Complementation of pimT}

In order to complement the $\Delta$ pim $T$ replacement mutant, a 924 bp DNA fragment containing the entire pimT gene including its own promoter was amplified by PCR with primers Ft (5'-GAATTCGTGTGTCCAGGTGACCGTC-3') and Rt (5'-CGTGAATTCGATGCCGAGCGC-3'). The PCR product was digested with EcoRI and ligated into an EcoRIcut pSET152neo $\left(\mathrm{Am}^{\mathrm{R}}, \mathrm{Km}^{\mathrm{R}}\right.$, pUC18 replicon, ФC31 $a t t P)$, to yield pSETpimT. This plasmid was then transferred by conjugation from E. coli ET12567 [pUZ8002] to the $S$. natalensis $\triangle \mathrm{PimT}$ mutant as previously described [50].

\section{Promoter activity assessment}

To assess the activity of the putative pimT promoter a 143 bp DNA fragment upstream from the ATG start codon was amplified by PCR using primers (5'-GGATCCGTGCCCGTCACCTCC-3') and (5'-GAATTCGTGTGTCCAGGTGACCGTC-3'). The PCR product was digested with BamHI and EcoRI and cloned in the same sites of pIJ4083 to yield pPpimT, which was subsequently transformed into S. lividans 66 [44]. For promoter activity studies approximately $1 \times 10^{6} \mathrm{~S}$. lividans spores were pre-germinated in $2 \times$ YT liquid medium for $8 \mathrm{~h}$ at $30^{\circ} \mathrm{C}$. Germinated spores were harvested by centrifugation, resuspended in YEME medium and used to inoculate 100 $\mathrm{ml}$ of the same medium. Thiostrepton $(5 \mu \mathrm{g} / \mathrm{ml})$ and kanamycin $(50 \mu \mathrm{g} / \mathrm{ml})$ were added as selective antibiotics. Catechol 2,3-dioxygenase activity of the $x y l E$ reporter gene was measured as described by Kieser et al.[44].

\section{Primer extension}

Total RNA was isolated from S. lividans harbouring pPpimT as described [51]. For primer extension experiments, $16 \mu \mathrm{g}$ RNA was hybridized to 20 pmol 6FAMlabelled primer (5'-CACATGGCCCGGTCGCATTACAC$\left.3^{\prime}\right)$ complementary to the 5'-coding region of $x y l E$. Reverse transcription was carried out with Superscript III (Invitrogen), and the products loaded into an ABI 3130 DNA genetic analyzer and analysed with the Gene Mapper ${ }^{\circledast}$ (Applied Biosystems) program.

\section{Isolation of total RNA}

S. natalensis ATCC 27448 was grown for $48 \mathrm{~h}$ in YEME medium (stationary phase of growth), the cultures were then mixed with one volume $40 \%(\mathrm{v} / \mathrm{v})$ glycerol, and mycelia were harvested by centrifugation and immediately frozen by immersion in liquid nitrogen. Frozen mycelium was then broken by shearing in a mortar, and the frozen lysate was added to buffer RLT (Qiagen) in the presence of $1.5 \%(\mathrm{v} / \mathrm{v}) \beta$-mercaptoethanol. RNeasy Mini Spin columns were used for RNA isolation according to manufacturer's instructions. RNA preparations were treated with DNase I RNase-free (Promega) in order to eliminate possible chromosomal DNA contamination.

\section{Gene expression analysis by RT-PCR}

Transcription was studied by using the SuperScript ${ }^{\mathrm{TM}}$ OneStep RT-PCR system with Platinum ${ }^{\circledR}$ Taq DNA polymerase (Invitrogen), using $20 \mathrm{ng}$ of total RNA as template. Conditions were as follows: first strand cDNA synthesis, $50^{\circ} \mathrm{C}$ for $30 \mathrm{~min}$ followed by heating at $94^{\circ} \mathrm{C}$ for $2 \mathrm{~min}$; amplification, 28 or 30 cycles of $98^{\circ} \mathrm{C}$ for $15 \mathrm{sec}, 64$ or $69^{\circ} \mathrm{C}$ (depending of the set of primers used) for $30 \mathrm{sec}$, and $72^{\circ} \mathrm{C}$ for $\left.1 \mathrm{~min}\right]$. Primers were designed to generate PCR products of approximately $500 \mathrm{bp}$. The primers used for the detection of pimT transcripts were: PIMTS, 5'GTCGTCGGCAACCTCATCGGCTCATAC-3'; and PIM- 
TAS, 5'-GCGCCCAGGCCCCACAGA-3'. The primers used to amplify a cDNA of the lysA gene (encoding diaminopimelate decarboxylase) were: LYSAS, 5'CGCCCGCCCACAGCAGGTCTTC-3'; and LYSAAS, 5'TGGGGGTGCATGAGGAACTGAT-3'. Negative controls were carried out with each set of primers and Platinum ${ }^{\circledast}$ Taq DNA polymerase in order to confirm the absence of contaminating DNA in the RNA preparations. The identity of each amplified product was corroborated by direct sequencing of the PCR product.

\section{Assay of pimaricin production}

To assay pimaricin in culture broths, $0.5 \mathrm{ml}$ of culture was extracted with $4 \mathrm{ml}$ of butanol, and the organic phase was diluted in water-saturated butanol to bring the absorbance at $319 \mathrm{~nm}$ in the range of 0.1 to 0.4 units. Control solutions of pure pimaricin (Sigma) were used as control. To confirm the identity of pimaricin, an UV-visible absorption spectrum (absorption peaks at 319, 304, 291 and $281 \mathrm{~nm}$ ) was routinely determined in a Hitachi U2900 spectrophotometer. Quantitative determination of pimaricin was performed as previously described [52].

\section{Determination of PI factor}

Quantitative determination of PI factor was carried out by reverse phase HPLC using a Waters 600 unit coupled to a PDA 996 detector equipped with a Polarity dC18 column $(3.9 \times 150 \mathrm{~mm}$; particle size, $5 \mu \mathrm{m})$ after derivatization with FMOC (fluorenylmethyl chloroformate) [53]. The PI factor elutes at a retention time of 9.3 min using a mobile phase mixture consisting of a gradient $(1.5 \mathrm{ml} / \mathrm{min})$ of acetonitrile in $50 \mathrm{mM}$ sodium acetate $\mathrm{pH} 4.2$ (acetonitrile concentration: $25 \%$ for $2 \mathrm{~min}$, up to $75 \% 2-13 \mathrm{~min}$, up to $100 \% 13-14 \mathrm{~min}, 100 \% 14-18 \mathrm{~min}$, down to $0 \% 18-19$ min, 0\% 19-23 min). Pure PI factor [30] was used as standard. Intracellular PI-factor was extracted as described elsewhere [52].

\section{Nucleotide sequence accession number}

The sequence reported here has been deposited in the GenBank database under the accession number $\underline{\text { FM864219. }}$.

\section{Competing interests}

The authors declare that they have no competing interests.

\section{Authors' contributions}

CMV created mutant strain, mapped the transcriptional start site and studied pimT expression. JSA and SMG carried out liquid culture experiments and assisted in PI-factor purification. TDP performed gene cloning and complementation experiments. JFM participated in the design of the study and assisted in manuscript writing. JFA conceived and supervised the study, reviewed results and drafted the manuscript. All authors have read and approved the final manuscript.

\section{Acknowledgements}

This work was supported by a Spanish Ministry of Science and Innovation Grant to J.F.A. (BIO2007-67585), and by a Grant from the Junta de Castilla y León (GRII 7). J.S-A. and T.D.P. were the recipients of F.P.U. fellowships from the Ministerio de Educación y Ciencia (AP2005-3644 and AP200702055 respectively). S.M.G. received a fellowship from the University of León. We thank Dr. Antonio Rodríguez-García for valuable help in the analysis of the promoter region, and J. Merino, B. Martín, and A. Casenave for excellent technical assistance.

\section{References}

I. Paulsen IT, Brown MH, Skurray RA: Proton-dependent multidrug efflux systems. Microbiol Rev 1996, 60:575-608.

2. Fuqua C, Winans SC, Greenberg EP: Census and consensus in bacterial ecosystems: the LuxR-Luxl family of quorum-sensing transcriptional regulators. Annu Rev Microbiol 1996, 50:727-75I.

3. Liu JY, Miller PF, Willard J, Olson ER: Functional and biochemical characterization of Escherichia coli sugar efflux transporters. J Biol Chem 1999, 274:22977-22984.

4. Krämer R: Secretion of amino acids by bacteria: physiology and mechanism. FEMS Microbiol Rev 1994, I3:75-94.

5. Eggeling L, Sahm H: New ubiquitous translocators: amino acid export by Corynebacterium glutamicum and Escherichia coli. Arch Microbiol 2003, 180:155-160.

6. Aleshin VV, Zakataeva NP, Livshits VA: A new family of aminoacid-efflux proteins. Trends Biochem Sci 1999, 24:133-I35.

7. Vrljiæ M, Sahm H, Eggeling L: A new type of transporter with a new type of cellular function: L-lysine export from Corynebacterium glutamicum. Mol Microbiol 1996, 22:8I5-826.

8. Bellmann A, Vrljiæ M, Pátek M, Sahm H, Krämer R, Eggeling L: Expression control and specificity of the basic amino acid exporter LysE of Corynebacterium glutamicum. Microbiology 2001, I47:1765-1774.

9. Zakataeva NP, Aleshin VV, Tokmakova IL, Troshin PV, Livshits VA: The novel transmembrane Escherichia coli proteins involved in the amino acid efflux. FEBS Lett I999, 452:228-232.

10. Franke I, Resch A, Dassler T, Maier T, Böck A: YfiK from Escherichia coli promotes export of O-acetylserine and cysteine. J Bacteriol 2003, I 85: I I6I-I I66.

II. Kutukova EA, Livshits VA, Altman IP, Ptitsyn LR, Zyiatdinov MH, Tokmakova IL, Zakataeva NP: The yeaS (leuE) gene of Escherichia coli encodes an exporter of leucine, and the Lrp protein regulates its expression. FEBS Lett 2005, 579:4629-4634.

12. Yen M-R, Tseng Y-H, Simic P, Sahm H, Eggeling L, Saier MH: The ubiquitous ThrE family of putative transmembrane amino acid efflux transporters. Res Microbiol 2002, I 53:19-25.

13. Simic P, Sahm H, Eggeling L: L-threonine export: use of peptides to identify a new translocator from Corynebacterium glutamicum. J Bacteriol 200I, I 83:5317-5324.

14. Dassler T, Maier T, Winterhalter C, Böck A: Identification of a major facilitator protein from Escherichia coli involved in efflux of metabolites of the cysteine pathway. Mol Microbiol 2000, 36: II0I-III2.

15. Liras P, Martín JF: $\beta$-Lactam Antibiotics. In Encyclopedia of Microbiology Third edition. Edited by: Schaechter M. Oxford: Elsevier Ltd., Oxford; 2009:274-289.

16. Martín JF: Phosphate control of the biosynthesis of antibiotics and other secondary metabolites is mediated by the PhoRPhoP system: An unfinished story. J Bacteriol 2004, I 86:5197-5201.

17. Bibb MJ: Regulation of secondary metabolism in Streptomycetes. Curr Opin Microbiol 2005, 8:208-2I5.

18. Aparicio JF, Mendes MV, Antón N, Recio E, Martín JF: Polyene macrolide antibiotic biosynthesis. Curr Med Chem 2004, I I: | 645-1656.

19. Caffrey P, Aparicio JF, Malpartida F, Zotchev SB: Biosynthetic engineering of polyene macrolides towards generation of 
improved antifungal and antiparasitic agents. Curr Top Med Chem 2008, 8:639-653.

20. Aparicio JF, Colina AJ, Ceballos E, Martín JF: The biosynthetic gene cluster for the 26-membered ring polyene macrolide pimaricin: A new polyketide synthase organization encoded by two subclusters separated by functionalization genes. J Biol Chem 1999, 274:10133-10139.

21. Aparicio JF, Fouces R, Mendes MV, Olivera N, Martín JF: A complex multienzyme system encoded by five polyketide synthase genes is involved in the biosynthesis of the 26-membered polyene macrolide pimaricin in Streptomyces natalensis. Chem Biol 2000, 7:895-905.

22. Mendes MV, Recio E, Fouces R, Luiten R, Martin JF, Aparicio JF: Engineered biosynthesis of novel polyenes: a pimaricin derivative produced by targeted gene disruption in Streptomyces natalensis. Chem Biol 200I, 8:635-644.

23. Mendes MV, Antón N, Martín JF, Aparicio JF: Characterization of the polyene macrolide P450 epoxidase from Streptomyces natalensis that converts deepoxypimaricin into pimaricin. Biochem J 2005, 386:57-62.

24. Mendes MV, Recio E, Antón N, Guerra SM, Santos-Aberturas J, Martín JF, Aparicio JF: Cholesterol oxidases act as signalling proteins for the biosynthesis of the polyene macrolide pimaricin. Chem Biol 2007, 14:279-290.

25. Antón N, Mendes MV, Martín JF, Aparicio JF: Identification of PimR as a positive regulator of pimaricin biosynthesis in Streptomyces natalensis. J Bacteriol 2004, 186:2567-2575.

26. Antón N, Santos-Aberturas J, Mendes MV, Guerra SM, Martín JF, Aparicio JF: PimM, a PAS domain positive regulator of pimaricin biosynthesis in Streptomyces natalensis. Microbiology 2007, I53(Pt 9):3174-3183.

27. Aparicio JF, Martín JF: Microbial cholesterol oxidase: Bioconversion enzymes or signal proteins? Mol Biosyst 2008, 4:804-809.

28. Martín JF, Aparicio JF: Enzymology of the Polyenes Pimaricin and Candicidin Biosynthesis. Methods Enzymol 2009, 459:215-242.

29. Horinouchi S, Beppu T: Autoregulatory factors and communication in actinomycetes. Annu Rev Microbiol 1992, 46:377-398.

30. Recio E, Colinas A, Rumbero A, Aparicio JF, Martín JF: PI factor, a novel type quorum-sensing inducer elicits pimaricin production in Streptomyces natalensis. J Biol Chem 2004, 279:41586-4I593.

31. Rascher A, Hu Z, Viswanathan N, Schirmer A, Reid R, Nierman WC, Lewis $M$, Hutchinson CR: Cloning and characterization of a gene cluster for geldanamycin production in Streptomyces hygroscopicus NRRL 3602. FEMS Microbiol Lett 2003, 21 8:223-230.

32. Oliynyk M, Samborskyy M, Lester JB, Mironenko T, Scott N, Dickens S, Haydock SF, Leadlay PF: Complete genome sequence of the erythromycin-producing bacterium Saccharopolyspora erythraea NRRL 2338. Nat Biotechnol 2007, 25:447-453.

33. Kruse D, Krämer R, Eggeling L, Rieping M, Pfefferle W, Tchieu JH, Chung YJ, Saier MH Jr, Burkovski A: Influence of threonine exporters on threonine production in Escherichia coli. Appl Microbiol Biotechnol 2002, 59:205-2I 0.

34. Burkovski A, Krämer R: Bacterial amino acid transport proteins: occurrence, functions, and significance for biotechnological applications. Appl Microbiol Biotechnol 2002, 58:265-274.

35. Baltz RH: Genetic manipulation of antibiotic-producing Streptomyces. Trends Microbiol 1998, 6:76-83.

36. Combes P, Till R, Bee S, Smith MC: The Streptomyces genome contains multiple pseudo-att $B$ sites for the $\Phi C 3 \mathrm{I}$-encoded site-specific recombination system. J Bacteriol 2002, I 84:5746-5752.

37. Zakataeva NP, Kutukova EA, Gronskii SV, Troshin PV, Livshits VA, Aleshin VV: Export of metabolites by the proteins of the DMT and RhtB families and its possible role in intercellular communication. Mikrobiologiia. 2006, 75(4):509-520.

38. Robson ND, Cox AR, McGowan SJ, Bycroft BW, Salmond GP: Bacterial $\mathbf{N}$-acyl-homoserine-lactone-dependent signalling and its potential biotechnological applications. Trends Biotechnol 1997, I 5:458-464.

39. Visick KL, Fuqua C: Decoding microbial chatter: cell-cell communication in bacteria. J Bacteriol 2005, 187:5507-55 I 9 .

40. Horinouchi S, Beppu T: A-factor as a microbial hormone that controls cellular differentiation and secondary metabolism in Streptomyces griseus. Mol Microbiol 1994, I 2:859-864.
4I. Lee KM, Lee CK, Choi SU, Park HR, Kitani S, Nihira T, Hwang YI: Cloning and in vivo functional analysis by disruption of a gene encoding the gamma-butyrolactone autoregulator receptor from Streptomyces natalensis. Arch Microbiol 2005, 184:249-257.

42. Van Houdt R, Aertsen A, Moons P, Vanoirbeek K, Michiels CW: Nacyl-L-homoserine lactone signal interception by Escherichia coli. FEMS Microbiol Lett 2006, 256:83-89.

43. Voeykova T, Emelyanova L, Tabakov V, Mkrtumyam N: Transfer of plasmid PTOI from Escherichia coli to various representatives of the order Actinomycetales by intergeneric conjugation. FEMS Microbiol Lett 1998, 162:47-52.

44. Kieser T, Bibb MJ, Buttner MJ, Chater KF, Hopwood DA: Practical Streptomyces genetics Norwich, John Innes Foundation; 2000.

45. Datsenko KA, Wanner BL: One-step inactivation of chromosomal genes in Escherichia coli K-1 2 using PCR products. Proc Natl Acad Sci USA 2000, 97:6640-6645.

46. Gust B, Challis GL, Fowler K, Kieser T, Chater KF: PCR-targeted Streptomyces gene replacement identifies a protein domain needed for biosynthesis of the sesquiterpene soil odor geosmin. Proc Natl Acad Sci USA 2003, 100: I54 I-I546.

47. Bierman M, Logan R, O'Brien K, Seno ET, Rao RN, Schoner BE: Plasmid cloning vectors for the conjugal transfer of DNA from Escherichia coli to Streptomyces spp. Gene 1992, I I 6:43-49.

48. Rodríguez-García A, Santamarta I, Pérez-Redondo R, Martín JF, Liras $P$ : Characterization of a two-gene operon epeRA involved in multidrug resistance in Streptomyces clavuligerus. Res Microbiol 2006, 157:559-568.

49. Sambrook J, Russell DW: Molecular cloning: a laboratory manual 3rd edition. New York: Cold Spring Harbor Laboratory Press; $200 \mathrm{I}$.

50. Enríquez LL, Mendes MV, Antón N, Tunca S, Guerra SM, Martín JF, Aparicio JF: An efficient gene transfer system for the pimaricin producer Streptomyces natalensis. FEMS Microbiol Lett 2006, 257:3।2-3I8.

5I. Mendes MV, Tunca S, Antón N, Recio E, Sola-Landa A, Aparicio JF, Martín JF: The two-component phoR-phoP system of Streptomyces natalensis: Inactivation or deletion of phoP reduces the negative phosphate regulation of pimaricin biosynthesis. Metab Eng 2007, 9:217-227.

52. Recio E, Aparicio JF, Rumbero A, Martín JF: Glycerol, ethylene glycol and propanediol elicit pimaricin biosynthesis in the PIfactor defective strain Streptomyces natalensis npi287 and increase polyene production in several wild type actinomycetes. Microbiology 2006, I 52:3 I47-3I 56.

53. Sim KL, Perry D: Analysis of swainsonine and its early metabolic precursors in cultures of Metarhizium anisopliae. Glycoconj J 1997, 14:661-668.
Publish with Bio Med Central and every scientist can read your work free of charge

"BioMed Central will be the most significant development for disseminating the results of biomedical research in our lifetime. "

Sir Paul Nurse, Cancer Research UK

Your research papers will be:

- available free of charge to the entire biomedical community

- peer reviewed and published immediately upon acceptance

- cited in PubMed and archived on PubMed Central

- yours - you keep the copyright
BioMedcentral 